\title{
Existence of Positive Solution to System of Nonlinear Third-Order Three-Point BVPs
}

\author{
Jian-Ping Sun, Xue-Mei Yang, and Ya-Hong Zhao \\ Department of Applied Mathematics, Lanzhou University of Technology, Lanzhou 730050, China \\ Correspondence should be addressed to Jian-Ping Sun; jpsun@lut.cn
}

Received 13 April 2016; Accepted 16 June 2016

Academic Editor: Enrique Llorens-Fuster

Copyright (C) 2016 Jian-Ping Sun et al. This is an open access article distributed under the Creative Commons Attribution License, which permits unrestricted use, distribution, and reproduction in any medium, provided the original work is properly cited.

We are concerned with the following system of third-order three-point boundary value problems: $u^{\prime \prime \prime}(t)+f(t, v(t))=0, t \in(0,1)$, $v^{\prime \prime \prime}(t)+g(t, u(t))=0, t \in(0,1), u(0)=u^{\prime \prime}(0)=0, u^{\prime}(1)=\alpha u(\eta), v(0)=v^{\prime \prime}(0)=0$, and $v^{\prime}(1)=\alpha v(\eta)$, where $0<\eta<1$ and $0<\alpha<1 / \eta$. By imposing some suitable conditions on $f$ and $g$, we obtain the existence of at least one positive solution to the above system. The main tool used is the theory of the fixed-point index.

\section{Introduction}

Third-order differential equations arise from a variety of different areas of applied mathematics and physics, for example, in the deflection of a curved beam having a constant or varying cross section, a three-layer beam, electromagnetic waves, or gravity driven flows, and so on [1].

Recently, there are a lot of papers concerning the existence of positive solutions to third-order three-point boundary value problems (BVPs for short); see [2-13] and the references therein. Yet, only in a few papers has the problem of existence of positive solutions to systems of third-order three-point BVPs been considered.

It is worth mentioning that there are some excellent works on systems of second-order or higher-order multipoint BVPs; see [14-18].

In this paper, we study the existence of positive solution for the following system of nonlinear third-order three-point BVPs:

$$
\begin{aligned}
& v(0)=v^{\prime \prime}(0)=0, \\
& v^{\prime}(1)=\alpha v(\eta) .
\end{aligned}
$$

Throughout this paper, we always assume that the following conditions are fulfilled:

$$
\begin{aligned}
& \left(H_{1}\right) 0<\eta<1 \text { and } 0<\alpha<1 / \eta \text {. } \\
& \left(H_{2}\right) f, g \in C([0,1] \times[0, \infty),[0, \infty)) \text { and } f(t, 0) \equiv 0 \text {, } \\
& g(t, 0) \equiv 0, t \in[0,1] \text {. }
\end{aligned}
$$

If $(u, v) \in C^{3}([0,1],[0, \infty)) \times C^{3}([0,1],[0, \infty))$ satisfies the differential equations and boundary conditions in system (1), then $(u, v)$ is said to be a solution of system (1). If $(u, v)$ is a solution of system (1) and $u(t)>0, v(t)>0, t \in(0,1)$, then $(u, v)$ is said to be a positive solution of system (1).

To end this section, we state the following results on the theory of the fixed-point index [19].

Let $E$ be a real Banach space, $P \subset E$ a cone, and $\theta$ the zero element in $E$. For $\rho>0$, we denote

$$
B_{\rho}=\{u \in E:\|u\|<\rho\} .
$$

Theorem 1. Let $A: \overline{B_{\rho}} \cap P \rightarrow P$ be a completely continuous operator. If there exists $u_{0} \in P \backslash\{\theta\}$ such that

$$
\begin{aligned}
& u(0)=u^{\prime \prime}(0)=0, \\
& u^{\prime}(1)=\alpha u(\eta),
\end{aligned}
$$

$$
u-A u \neq \lambda u_{0}, \quad u \in \partial B_{\rho} \cap P, \lambda \geq 0,
$$


Theorem 2. Let $A: \overline{B_{\rho}} \cap P \rightarrow P$ be a completely continuous operator which has no fixed point on $\partial B_{\rho} \cap P$. If $\|A u\| \leq\|u\|$ for all $u \in \partial B_{\rho} \cap P$, then $i\left(A, B_{\rho} \cap P, P\right)=1$.

\section{Preliminaries}

Let $E=C[0,1]$ be equipped with the maximum norm. Then $E$ is a Banach space.

Lemma 3 (see [7]). For any $y \in E$, the BVP

$$
\begin{aligned}
u^{\prime \prime \prime}(t)+y(t) & =0, \quad t \in(0,1), \\
u(0) & =u^{\prime \prime}(0)=0, \\
u^{\prime}(1) & =\alpha u(\eta)
\end{aligned}
$$

has a unique solution

$$
u(t)=\int_{0}^{1} G(t, s) y(s) d s, \quad t \in[0,1],
$$

where

$$
\begin{aligned}
& G(t, s)=\frac{1}{2(1-\alpha \eta)} \\
& \quad . \begin{cases}2 t-(1-\alpha \eta)\left(t^{2}+s^{2}\right)-\alpha t\left(\eta^{2}+s^{2}\right), & s \leq \min \{\eta, t\}, \\
2 t-t^{2}-s^{2}+\alpha \eta(t-s)^{2}, & \eta \leq s \leq t, \\
2 t-2 t s(1-\alpha \eta)-\alpha t\left(\eta^{2}+s^{2}\right), & t \leq s \leq \eta, \\
2 t(1-s), & s \geq \max \{\eta, t\} .\end{cases}
\end{aligned}
$$

For convenience, we denote

$$
\begin{aligned}
& a=\frac{1+\alpha \eta}{1-\alpha \eta}, \\
& \gamma=\frac{\eta^{2}}{2(1+\alpha \eta)} \min \{1, \alpha\} .
\end{aligned}
$$

Obviously, $a>0$ and $0<\gamma<1$.

Lemma 4 (see [7]). For any $(t, s) \in[0,1] \times[0,1], 0 \leq G(t, s) \leq$ $a(1-s)$.

Lemma 5. For any $(t, s) \in[\eta, 1] \times[0,1], G(t, s) \geq \gamma a(1-s)$.

Proof. Since $G(t, 1)=0$, we only need to consider $(t, s) \in$ $[\eta, 1] \times[0,1)$.

If $s \leq \eta \leq t$, then

$$
\begin{aligned}
\frac{G(t, s)}{a(1-s)} & =\frac{2 t-(1-\alpha \eta)\left(t^{2}+s^{2}\right)-\alpha t\left(\eta^{2}+s^{2}\right)}{2(1+\alpha \eta)(1-s)} \\
& >\frac{2 t-(1-\alpha \eta)(t+t s)-\alpha t(\eta+\eta s)}{2(1+\alpha \eta)(1-s)} \\
& =\frac{t}{2(1+\alpha \eta)} \geq \frac{\eta}{2(1+\alpha \eta)}>\frac{\eta^{2}}{2(1+\alpha \eta)} \\
& \geq \gamma .
\end{aligned}
$$

If $\eta \leq s \leq t$, then

$$
\begin{aligned}
\frac{G(t, s)}{a(1-s)} & =\frac{2 t-t^{2}-s^{2}+\alpha \eta(t-s)^{2}}{2(1+\alpha \eta)(1-s)} \\
& \geq \frac{2 t-2 t(1-\alpha \eta)-2 \alpha \eta t s}{2(1+\alpha \eta)(1-s)} \geq \frac{\alpha \eta^{2}}{1+\alpha \eta} \\
& >\frac{\alpha \eta^{2}}{2(1+\alpha \eta)} \geq \gamma .
\end{aligned}
$$

If $\eta \leq t \leq s$, then

$$
\frac{G(t, s)}{a(1-s)}=\frac{t}{1+\alpha \eta} \geq \frac{\eta}{1+\alpha \eta}>\frac{\eta^{2}}{2(1+\alpha \eta)} \geq \gamma .
$$

Therefore, for any $(t, s) \in[\eta, 1] \times[0,1], G(t, s) \geq \gamma a(1-s)$.

Lemma 6. Let $y \in E$ and $y(t) \geq 0, t \in[0,1]$. Then the unique solution $u$ of $B V P(4)$ satisfies

$$
\begin{aligned}
u(t) & \geq 0, \quad t \in[0,1], \\
\min _{t \in[\eta, 1]} u(t) & \geq \eta\|u\| .
\end{aligned}
$$

Proof. Since $y(t) \geq 0, t \in[0,1]$, it follows from Lemma 4 that $u(t) \geq 0$ for $t \in[0,1]$. In view of $u^{\prime \prime \prime}(t)=-y(t) \leq 0$, $t \in(0,1)$, we have $u^{\prime \prime}(t) \leq u^{\prime \prime}(0)=0, t \in(0,1)$. This shows that $u^{\prime}(t) \geq u^{\prime}(1)=\alpha u(\eta) \geq 0, t \in(0,1)$, which indicates that

$$
\begin{aligned}
\min _{t \in[\eta, 1]} u(t) & =u(\eta), \\
\|u\| & =u(1) .
\end{aligned}
$$

On the other hand, it follows from the fact $u^{\prime \prime}(t) \leq 0$ for $t \in$ $(0,1)$ that $u$ is concave down on $[0,1]$, which together with $u(0)=0$ implies that $u(\eta) \geq \eta u(1)$. In view of (12), we get that $\min _{t \in[\eta, 1]} u(t) \geq \eta\|u\|$.

Lemma 7. There exists $x_{0} \in(0,(4 \sqrt{3} / 9) \pi)$ such that

$$
\begin{aligned}
x_{0} e^{-x_{0}} & +\alpha e^{-\eta x_{0}}+2 x_{0} e^{(1 / 2) x_{0}} \cos \frac{\sqrt{3}}{2} x_{0} \\
- & 2 \alpha e^{(1 / 2) \eta x_{0}} \sin \left(\frac{\pi}{6}+\frac{\sqrt{3}}{2} \eta x_{0}\right)=0 .
\end{aligned}
$$

Proof. Let

$$
\begin{aligned}
\varphi(x)=x e^{-x}+\alpha e^{-\eta x}+2 x e^{(1 / 2) x} \cos \frac{\sqrt{3}}{2} x & \\
-2 \alpha e^{(1 / 2) \eta x} \sin \left(\frac{\pi}{6}+\frac{\sqrt{3}}{2} \eta x\right), & \\
x & \in\left[0, \frac{4 \sqrt{3}}{9} \pi\right] .
\end{aligned}
$$

Then it is obvious that

$$
\begin{aligned}
\varphi(0) & =0, \\
\varphi^{\prime}(0) & =3(1-\alpha \eta)>0 .
\end{aligned}
$$


This indicates that there exists $x^{*} \in(0,(4 \sqrt{3} / 9) \pi)$ such that $\varphi\left(x^{*}\right)>0$, which together with the fact that

$$
\begin{aligned}
& \varphi\left(\frac{4 \sqrt{3}}{9} \pi\right)=\frac{4 \sqrt{3}}{9} \pi e^{-(4 \sqrt{3} / 9) \pi}+\alpha e^{-(4 \sqrt{3} / 9) \pi \eta}-\frac{4 \sqrt{3}}{9} \\
& \cdot \pi e^{(2 \sqrt{3} / 9) \pi}-2 \alpha e^{(2 \sqrt{3} / 9) \pi \eta} \sin \left(\frac{\pi}{6}+\frac{2 \pi}{3} \eta\right)<\frac{4 \sqrt{3}}{9} \\
& \cdot \pi e^{-(4 \sqrt{3} / 9) \pi}+\alpha e^{-(4 \sqrt{3} / 9) \pi \eta}-\frac{4 \sqrt{3}}{9} \pi e^{(2 \sqrt{3} / 9) \pi} \\
& -\alpha e^{(2 \sqrt{3} / 9) \pi \eta}=-\left[\frac{4 \sqrt{3}}{9} \pi\left(e^{(2 \sqrt{3} / 9) \pi}-e^{-(4 \sqrt{3} / 9) \pi}\right)\right. \\
& \left.+\alpha\left(e^{(2 \sqrt{3} / 9) \pi \eta}-e^{-(4 \sqrt{3} / 9) \pi \eta}\right)\right]<0
\end{aligned}
$$

implies that there exists $x_{0} \in\left(x^{*},(4 \sqrt{3} / 9) \pi\right) \subset(0,(4 \sqrt{3} / 9) \pi)$ such that $\varphi\left(x_{0}\right)=0$; that is, (13) is satisfied.

In the remainder of this paper, we always assume that $x_{0}$ is defined as in Lemma 7 and $u_{0}(t)=-e^{-x_{0} t}+2 e^{(1 / 2) x_{0} t} \sin (\pi / 6+$ $\left.(\sqrt{3} / 2) x_{0} t\right), t \in[0,1]$.

Corollary 8. $x_{0}^{3}$ is an eigenvalue of the eigenvalue problem

$$
\begin{aligned}
u^{\prime \prime \prime}(t)+\lambda u(t) & =0, \quad t \in(0,1), \\
u(0) & =u^{\prime \prime}(0)=0, \\
u^{\prime}(1) & =\alpha u(\eta)
\end{aligned}
$$

and $u_{0}$ is an eigenfunction corresponding to the eigenvalue $x_{0}^{3}$.

Proof. By Lemma 7, it is obvious.

Let

$$
\begin{gathered}
P=\{u \in E: u(t) \geq 0, t \in[0,1]\}, \\
P_{0}=\left\{u \in P: \min _{t \in[\eta, 1]} u(t) \geq \eta\|u\|\right\} .
\end{gathered}
$$

Then $P$ and $P_{0}$ are cones in $E$. Now, we define a linear operator $B$ as follows:

$$
(B u)(t)=\int_{0}^{1} G(t, s) u(s) d s, \quad u \in P, t \in[0,1] .
$$

Lemma 9. Consider $B: P \rightarrow P_{0}$.

Proof. In view of Lemmas 3, 4, and 6, it is not difficult to verify that $B(P) \subset P_{0}$.

Lemma 10. Consider $u_{0} \in P$ and

$$
u_{0}(t)=x_{0}^{3}\left(B u_{0}\right)(t), \quad t \in[0,1] .
$$

Proof. Obviously, $u_{0}$ is continuous and $u_{0}(t) \geq e^{-x_{0} t}\left(e^{(3 / 2) x_{0} t}\right.$ $1) \geq 0, t \in[0,1]$, which indicates that $u_{0} \in P$. And it follows from Lemma 3 and Corollary 8 that $(20)$ is satisfied.

\section{Main Results}

Obviously, $(u, v)$ is a solution of system (1) if and only if $(u, v) \in C([0,1],[0, \infty)) \times C([0,1],[0, \infty))$ is a solution of the following system:

$$
\begin{aligned}
& u(t)=\int_{0}^{1} G(t, s) f(s, v(s)) d s, \quad t \in[0,1], \\
& v(t)=\int_{0}^{1} G(t, s) g(s, u(s)) d s, \quad t \in[0,1] .
\end{aligned}
$$

Moreover, system (21) can be written as the integral equation:

$$
\begin{array}{r}
u(t)=\int_{0}^{1} G(t, s) f\left(s, \int_{0}^{1} G(s, \tau) g(\tau, u(\tau)) d \tau\right) d s \\
t \in[0,1]
\end{array}
$$

If we define an operator $A$ on $P$ by

$$
\begin{array}{r}
(A u)(t) \\
=\int_{0}^{1} G(t, s) f\left(s, \int_{0}^{1} G(s, \tau) g(\tau, u(\tau)) d \tau\right) d s, \\
t \in[0,1],
\end{array}
$$

then it is easy to verify that $A: P \rightarrow P$ is completely continuous. Moreover, if $u \in P$ is a fixed point of $A$ and $v(t)=\int_{0}^{1} G(t, s) g(s, u(s)) d s, t \in[0,1]$, then $(u, v)$ is a solution of system (1).

Theorem 11. Assume that the following conditions are fulfilled:

$\left(A_{1}\right)$ There exists a constant $p \in(0,1]$ such that

$$
\begin{aligned}
& \text { (1) } \lim _{x \rightarrow \infty} \frac{f(t, x)}{x^{p}}>0, \\
& \text { (2) } \lim _{x \rightarrow \infty} \frac{g(t, x)}{x^{1 / p}}=\infty
\end{aligned}
$$

uniformly on $[0,1]$.

$\left(A_{2}\right)$ There exists a constant $q \in(0, \infty)$ such that

$$
\begin{aligned}
& \text { (1) } \lim _{x \rightarrow 0^{+}} \frac{f(t, x)}{x^{q}}<\infty, \\
& \text { (2) } \lim _{x \rightarrow 0^{+}} \frac{g(t, x)}{x^{1 / q}}=0
\end{aligned}
$$

uniformly on $[0,1]$.

Then system (1) has at least one positive solution.

Proof. First, let

$$
M=\left\{u \in P: u-A u=\lambda u_{0}, \lambda \geq 0\right\} .
$$

Then we may assert that $M$ is a bounded subset of $E$. 
In fact, if $u \in M$, then there exists $\lambda \geq 0$ such that $u=$ $A u+\lambda u_{0}$, which together with Lemma 10 implies that

$$
\begin{aligned}
u(t)= & (A u)(t)+\lambda u_{0}(t) \\
= & \int_{0}^{1} G(t, s) f\left(s, \int_{0}^{1} G(s, \tau) g(\tau, u(\tau)) d \tau\right) d s \\
& +\lambda x_{0}^{3}\left(B u_{0}\right)(t) \\
= & \int_{0}^{1} G(t, s)(F u)(s) d s+\lambda x_{0}^{3}\left(B u_{0}\right)(t) \\
= & B\left[(F u)(t)+\lambda x_{0}^{3} u_{0}(t)\right], \quad t \in[0,1]
\end{aligned}
$$

where $F: P \rightarrow P$ is defined as follows:

$$
(F u)(t)=f\left(t, \int_{0}^{1} G(t, s) g(s, u(s)) d s\right),
$$

$$
t \in[0,1] .
$$

In view of (27) and Lemma 9, we have $u \in P_{0}$. This implies that

$$
\|u\| \leq \frac{1}{\eta} \min _{t \in[\eta, 1]} u(t)
$$

On the other hand, from (1) of $\left(A_{1}\right)$, we know that there exist positive constants $C_{1}$ and $C_{2}$ such that

$$
f(t, x) \geq C_{1} x^{p}-C_{2}, \quad(t, x) \in[0,1] \times[0, \infty),
$$

which together with Jensen inequality and Lemma 4 implies that

$$
\begin{aligned}
& (A u)(t)=\int_{0}^{1} G(t, s) \\
& \cdot f\left(s, \int_{0}^{1} G(s, \tau) g(\tau, u(\tau)) d \tau\right) d s \\
& \geq \int_{0}^{1} G(t, s) \\
& \cdot\left[C_{1}\left(\int_{0}^{1} G(s, \tau) g(\tau, u(\tau)) d \tau\right)^{p}-C_{2}\right] d s \\
& \geq C_{1} \int_{0}^{1} G(t, s) \int_{0}^{1}(G(s, \tau))^{p}(g(\tau, u(\tau)))^{p} d \tau d s \\
& -C_{2} \int_{0}^{1} G(t, s) d s \geq C_{1} \int_{0}^{1} G(t, s) \\
& \cdot \int_{0}^{1}(G(s, \tau))^{p}(g(\tau, u(\tau)))^{p} d \tau d s-C_{3},
\end{aligned}
$$$$
t \in[0,1],
$$

where $C_{3}=(1 / 2) a C_{2}$.
From (2) of $\left(A_{1}\right)$, we know that there exists $C_{4}>0$ such that

$$
\begin{aligned}
(g(t, x))^{p} \geq \frac{2}{C_{1} \gamma^{p} a^{p-1} \eta m_{1}^{2}} x-C_{4}, & \\
& (t, x) \in[0,1] \times[0, \infty),
\end{aligned}
$$

where $m_{1}=\min _{t \in[\eta, 1]} \int_{\eta}^{1} G(t, s) d s>0$.

So, it follows from (31), (32), and Lemmas 4 and 5 that

$$
\begin{gathered}
u(t)=(A u)(t)+\lambda u_{0}(t) \geq(A u)(t) \geq C_{1} \int_{0}^{1} G(t, s) \\
\cdot \int_{0}^{1}(G(s, \tau))^{p}(g(\tau, u(\tau)))^{p} d \tau d s-C_{3}
\end{gathered}
$$$$
\geq C_{1} \int_{\eta}^{1} G(t, s) \int_{0}^{1}(G(s, \tau))^{p}(g(\tau, u(\tau)))^{p} d \tau d s
$$$$
-C_{3} \geq C_{1} \gamma^{p} a^{p} \int_{\eta}^{1} G(t, s)
$$$$
\cdot \int_{0}^{1}(1-\tau)^{p}(g(\tau, u(\tau)))^{p} d \tau d s-C_{3}
$$$$
\geq C_{1} \gamma^{p} a^{p} \int_{\eta}^{1} G(t, s) \int_{0}^{1}(1-\tau)(g(\tau, u(\tau)))^{p} d \tau d s
$$

$-C_{3} \geq C_{1} \gamma^{p} a^{p-1} \int_{\eta}^{1} G(t, s)$

$\cdot \int_{0}^{1} G(s, \tau)(g(\tau, u(\tau)))^{p} d \tau d s-C_{3}$

$\geq C_{1} \gamma^{p} a^{p-1} \int_{\eta}^{1} G(t, s)$

$\cdot \int_{0}^{1} G(s, \tau)\left[\frac{2}{C_{1} \gamma^{p} a^{p-1} \eta m_{1}^{2}} u(\tau)-C_{4}\right] d \tau d s$

$-C_{3}=\frac{2}{\eta m_{1}^{2}} \int_{\eta}^{1} G(t, s) \int_{0}^{1} G(s, \tau) u(\tau) d \tau d s$

$-C_{1} C_{4} \gamma^{p} a^{p-1} \int_{\eta}^{1} G(t, s) \int_{0}^{1} G(s, \tau) d \tau d s-C_{3}$

$=\frac{2}{\eta m_{1}^{2}} \int_{\eta}^{1} G(t, s)(B u)(s) d s$

$-\left[C_{1} C_{4} \gamma^{p} a^{p-1} \int_{\eta}^{1} G(t, s) \int_{0}^{1} G(s, \tau) d \tau d s+C_{3}\right]$

$$
\geq \frac{2}{\eta m_{1}^{2}} \min _{s \in[\eta, 1]}(B u)(s) \int_{\eta}^{1} G(t, s) d s
$$




$$
\begin{aligned}
& -\left[C_{1} C_{4} \gamma^{p} a^{p} \int_{\eta}^{1}(1-s) \int_{0}^{1} G(s, \tau) d \tau d s+C_{3}\right] \\
& \geq \frac{2}{\eta m_{1}} \min _{s \in[\eta, 1]}(B u)(s)-C_{5}, \quad t \in[\eta, 1],
\end{aligned}
$$

where $C_{5}=C_{1} C_{4} \gamma^{p} a^{p} \int_{\eta}^{1}(1-s) \int_{0}^{1} G(s, \tau) d \tau d s+C_{3}>0$.

Since $B(u) \in P_{0}$, we have

$$
\begin{aligned}
\min _{s \in[\eta, 1]}(B u)(s) & \geq \eta\|B u\| \geq \eta \int_{0}^{1} G(\eta, \tau) u(\tau) d \tau \\
& \geq \eta \int_{\eta}^{1} G(\eta, \tau) u(\tau) d \tau \\
& \geq \eta \min _{\tau \in[\eta, 1]} u(\tau) \int_{\eta}^{1} G(\eta, \tau) d \tau \\
& \geq \eta m_{1} \min _{\tau \in[\eta, 1]} u(\tau) .
\end{aligned}
$$

In view of (33) and (34), we get

$$
u(t) \geq 2 \min _{\tau \in[\eta, 1]} u(\tau)-C_{5}, \quad t \in[\eta, 1] .
$$

So,

$$
\min _{t \in[\eta, 1]} u(t) \geq 2 \min _{t \in[\eta, 1]} u(t)-C_{5},
$$

and so,

$$
\min _{t \in[\eta, 1]} u(t) \leq C_{5}
$$

which together with (29) indicates that

$$
\|u\| \leq \frac{C_{5}}{\eta} .
$$

This shows that $M$ is a bounded subset of $E$. Therefore, there exists a sufficiently larger $R>1$ such that

$$
u-A u \neq \lambda u_{0}, \quad u \in \partial B_{R} \cap P, \lambda \geq 0 .
$$

So, it follows from Theorem 1 that

$$
i\left(A, B_{R} \cap P, P\right)=0 .
$$

Next, from (1) of $\left(A_{2}\right)$, we have

$$
b:=\sup \left\{\frac{f(t, x)}{x^{q}}:(t, x) \in[0,1] \times(0,1]\right\}<\infty,
$$

which together with $f(t, 0) \equiv 0, t \in[0,1]$, implies that

$$
f(t, x) \leq b x^{q}, \quad(t, x) \in[0,1] \times[0,1] .
$$

Let

$$
\varepsilon=\min \left\{\frac{1}{m_{2}},\left(\frac{1}{(b+1) m_{2}^{q+1}}\right)^{1 / q}\right\}
$$

where $m_{2}=\max _{t \in[0,1]} \int_{0}^{1} G(t, s) d s>0$. Then from $(2)$ of $\left(A_{2}\right)$ and $g(t, 0) \equiv 0, t \in[0,1]$, we know that there exists $r \in(0,1]$ such that

$$
g(t, x) \leq \varepsilon x^{1 / q}, \quad(t, x) \in[0,1] \times[0, r] .
$$

By (44), for any $u \in \partial B_{r} \cap P$, we have

$$
\begin{aligned}
& \int_{0}^{1} G(s, \tau) g(\tau, u(\tau)) d \tau \leq \varepsilon \int_{0}^{1} G(s, \tau)(u(\tau))^{1 / q} d \tau \\
& \quad \leq \varepsilon m_{2}\|u\|^{1 / q}(\leq 1), \quad s \in[0,1]
\end{aligned}
$$

which together with (42) shows that

$$
\begin{aligned}
& (A u)(t) \\
& =\int_{0}^{1} G(t, s) f\left(s, \int_{0}^{1} G(s, \tau) g(\tau, u(\tau)) d \tau\right) d s \\
& \leq b \int_{0}^{1} G(t, s)\left(\int_{0}^{1} G(s, \tau) g(\tau, u(\tau)) d \tau\right)^{q} d s \\
& \leq b \varepsilon^{q} m_{2}^{q}\|u\| \int_{0}^{1} G(t, s) d s \leq b \varepsilon^{q} m_{2}^{q+1}\|u\| \\
& <(b+1) \varepsilon^{q} m_{2}^{q+1}\|u\| \leq\|u\|, \quad t \in[0,1] .
\end{aligned}
$$

This indicates that $\|A u\|<\|u\|, u \in \partial B_{r} \cap P$. So, it follows from Theorem 2 that

$$
i\left(A, B_{r} \cap P, P\right)=1 .
$$

In view of (40) and (47), we get

$$
\begin{aligned}
& i\left(A,\left(B_{R} \backslash \overline{B_{r}}\right) \cap P, P\right) \\
& \quad=i\left(A, B_{R} \cap P, P\right)-i\left(A, B_{r} \cap P, P\right)=-1 .
\end{aligned}
$$

Therefore, $A$ has at least one fixed point $u \in P$ and $r<\|u\|<$ $R$. Let

$$
v(t)=\int_{0}^{1} G(t, s) g(s, u(s)) d s, \quad t \in[0,1] .
$$

Then $(u, v)$ is a solution of system (1).

Thirdly, we prove $u(t)>0$ for $t \in(0,1)$.

Suppose on the contrary that there exists $t_{0} \in(0,1)$ such that $u\left(t_{0}\right)=0$. Since $u^{\prime \prime \prime}(t)=-f(t, v(t)) \leq 0, t \in(0,1)$, we know that $u^{\prime \prime}(t) \leq u^{\prime \prime}(0)=0, t \in(0,1)$. So, $u(t)$ is concave down on $[0,1]$, which together with $u(0)=u\left(t_{0}\right)=0$ implies that $u(1)=0$. At the same time, it follows from $u^{\prime \prime}(t) \leq 0$, $t \in(0,1)$, that $u^{\prime}(t) \geq u^{\prime}(1)=\alpha u(\eta) \geq 0, t \in(0,1)$. So, $0=u(0) \leq u(t) \leq u(1)=0, t \in[0,1]$; that is, $u(t) \equiv 0$, $t \in[0,1]$, which contradicts with the fact that $\|u\|>r>0$. Therefore, $u(t)>0, t \in(0,1)$.

Finally, we verify $v(t)>0$ for $t \in(0,1)$.

First, we may assert that $\|v\|>0$. Indeed, if $v(t) \equiv 0, t \in$ $[0,1]$, in view of $f(t, 0) \equiv 0, t \in[0,1]$, then we get

$$
\begin{aligned}
u(t) & =\int_{0}^{1} G(t, s) f(s, v(s)) d s \\
& =\int_{0}^{1} G(t, s) f(s, 0) d s=0, \quad t \in[0,1],
\end{aligned}
$$


which is a contradiction. This shows that $\|v\|>0$. So, by using the similar method to prove $u(t)>0$ for $t \in(0,1)$, we may obtain $v(t)>0, t \in(0,1)$.

To sum it up, $(u, v)$ is a positive solution of system (1).

\section{Competing Interests}

The authors declare that there is no conflict of interests regarding the publication of this paper.

\section{References}

[1] M. Greguš, Third Order Linear Differential Equations, vol. 22 of Mathematics and Its Applications (East European Series), D. Reidel, Dordrecht, The Netherlands, 1987.

[2] D. R. Anderson, "Green's function for a third-order generalized right focal problem," Journal of Mathematical Analysis and Applications, vol. 288, no. 1, pp. 1-14, 2003.

[3] Y. Sun, "Positive solutions of singular third-order three-point boundary value problem," Journal of Mathematical Analysis and Applications, vol. 306, no. 2, pp. 589-603, 2005.

[4] Z. Bai and X. Fei, "Existence of triple positive solutions for a third order generalized right focal problem," Mathematical Inequalities and Applications, vol. 9, no. 3, pp. 437-444, 2006.

[5] B. Yang, "Positive solutions of a third-order three-point boundary-value problem," Electronic Journal of Differential Equations, vol. 2008, no. 99, pp. 1-10, 2008.

[6] L.-J. Guo, J.-P. Sun, and Y.-H. Zhao, "Existence of positive solutions for nonlinear third-order three-point boundary value problems," Nonlinear Analysis: Theory, Methods and Applications, vol. 68, no. 10, pp. 3151-3158, 2008.

[7] J.-P. Sun, L.-J. Guo, and J.-G. Peng, "Multiple nondecreasing positive solutions for a singular third-order three-point BVP," Communications in Applied Analysis, vol. 12, no. 1, pp. 91-100, 2008.

[8] Q. Yao, "Positive solutions of singular third-order three-point boundary value problems," Journal of Mathematical Analysis and Applications, vol. 354, no. 1, pp. 207-212, 2009.

[9] Y. Sun, "Positive solutions for third-order three-point nonhomogeneous boundary value problems," Applied Mathematics Letters, vol. 22, no. 1, pp. 45-51, 2009.

[10] X. Feng, H. Feng, and D. Bai, "Eigenvalue for a singular thirdorder three-point boundary value problem," Applied Mathematics and Computation, vol. 219, no. 18, pp. 9783-9790, 2013.

[11] X.-L. Li, J.-P. Sun, and F.-D. Kong, "Existence of positive solution for a third-order three-point BVP with sign-changing Green's function," Electronic Journal of Qualitative Theory of Differential Equations, no. 30, pp. 1-11, 2013.

[12] F. J. Torres, "Positive solutions for a third-order three-point boundary-value problem," Electronic Journal of Differential Equations, vol. 2013, no. 147, pp. 1-11, 2013.

[13] J.-P. Sun and J. Zhao, "Iterative technique for a third-order three-point BVP with sign-changing Green's function," Electronic Journal of Differential Equations, vol. 2013, no. 215, pp. 1-9, 2013.

[14] Y. Zhou and Y. Xu, "Positive solutions of three-point boundary value problems for systems of nonlinear second order ordinary differential equations," Journal of Mathematical Analysis and Applications, vol. 320, no. 2, pp. 578-590, 2006.
[15] J. Henderson and R. Luca, "Positive solutions for a system of second-order multi-point boundary value problems," Applied Mathematics and Computation, vol. 218, no. 10, pp. 6083-6094, 2012.

[16] J. Henderson and R. Luca, "Positive solutions for a system of second-order nonlinear multi-point eigenvalue problems," Applied Mathematics and Computation, vol. 223, pp. 197-208, 2013.

[17] J. Henderson and R. Luca, "Positive solutions for systems of nonlinear second-order multipoint boundary value problems," Mathematical Methods in the Applied Sciences, vol. 37, no. 16, pp. 2502-2516, 2014.

[18] J. Henderson and R. Luca, "Positive solutions for a system of higher-order multi-point boundary value problems," Computers \& Mathematics with Applications, vol. 62, no. 10, pp. 39203932, 2011.

[19] H. Amann, "Fixed point equations and nonlinear eigenvalue problems in ordered Banach spaces," SIAM Review, vol. 18, no. 4, pp. 620-709, 1976. 


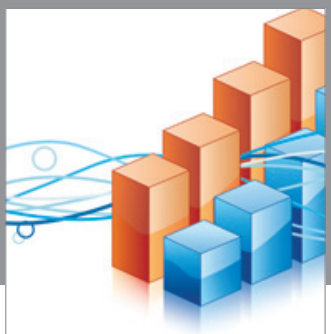

Advances in

Operations Research

vatem alat4

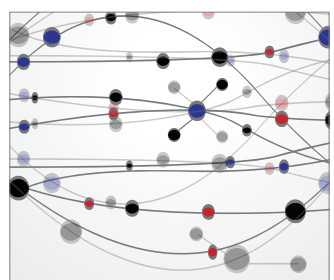

\section{The Scientific} World Journal
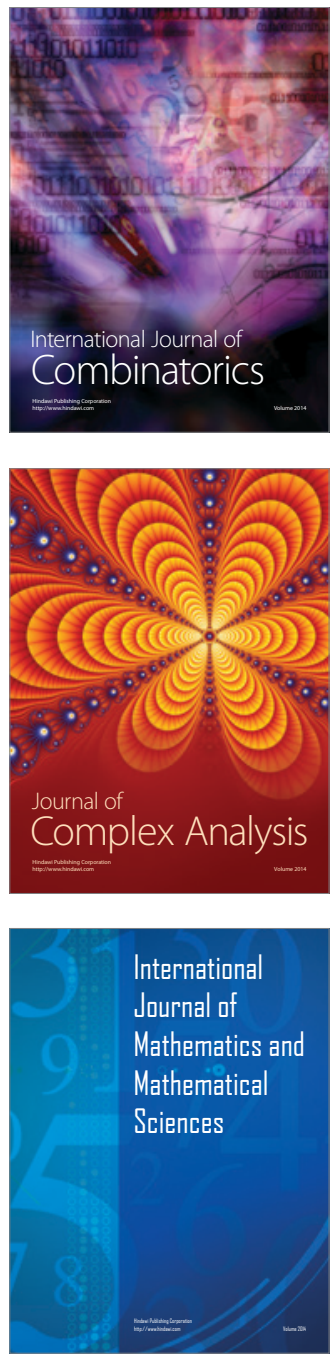
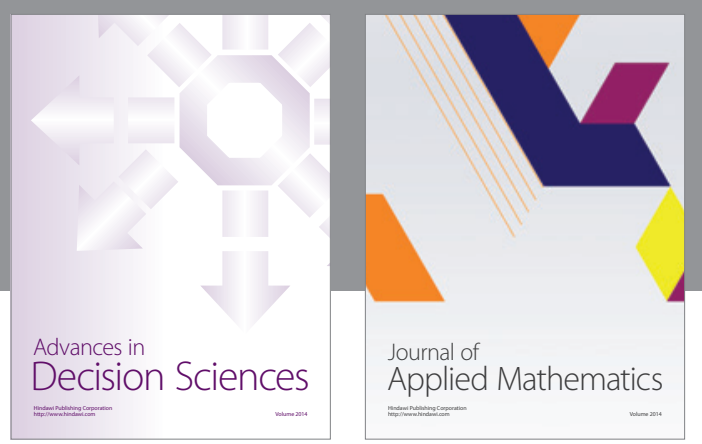

Algebra

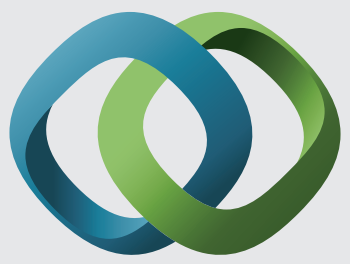

\section{Hindawi}

Submit your manuscripts at

http://www.hindawi.com
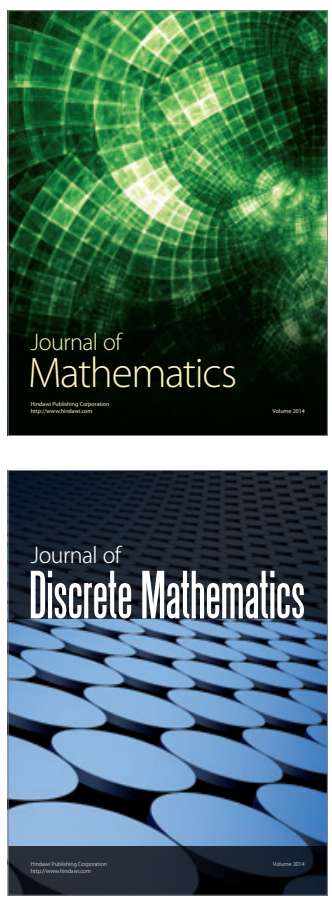

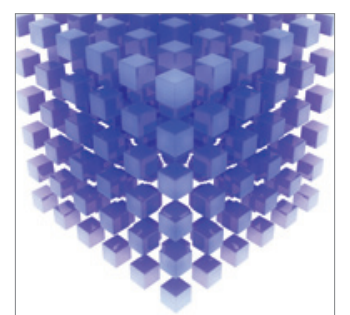

Mathematical Problems in Engineering
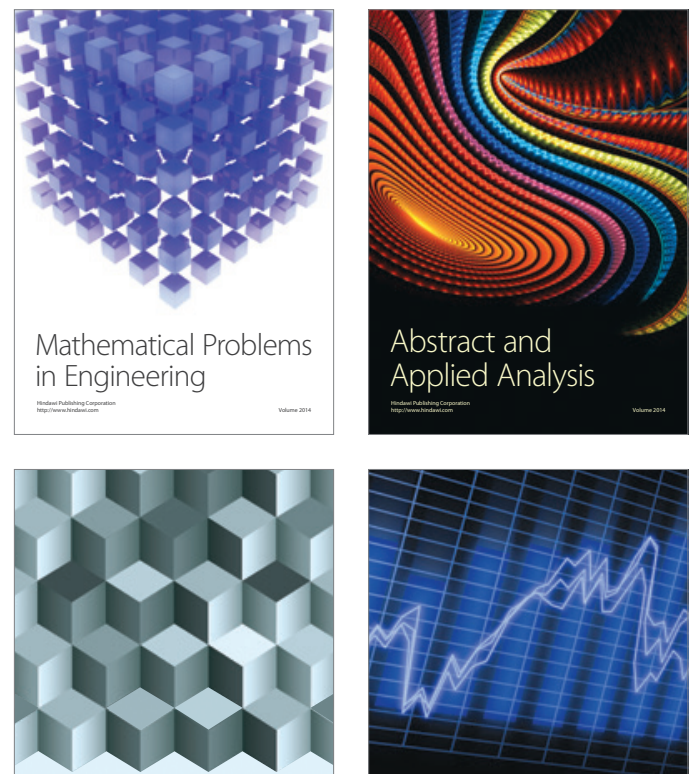

Journal of

Function Spaces

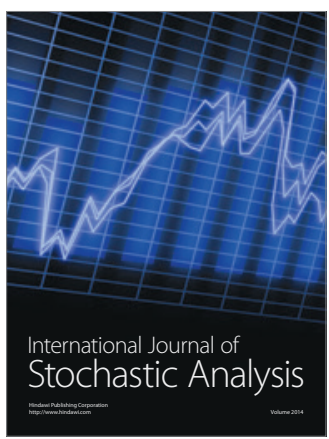

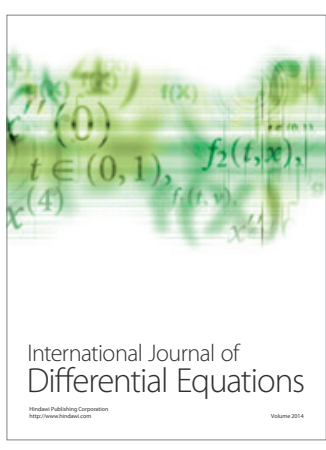
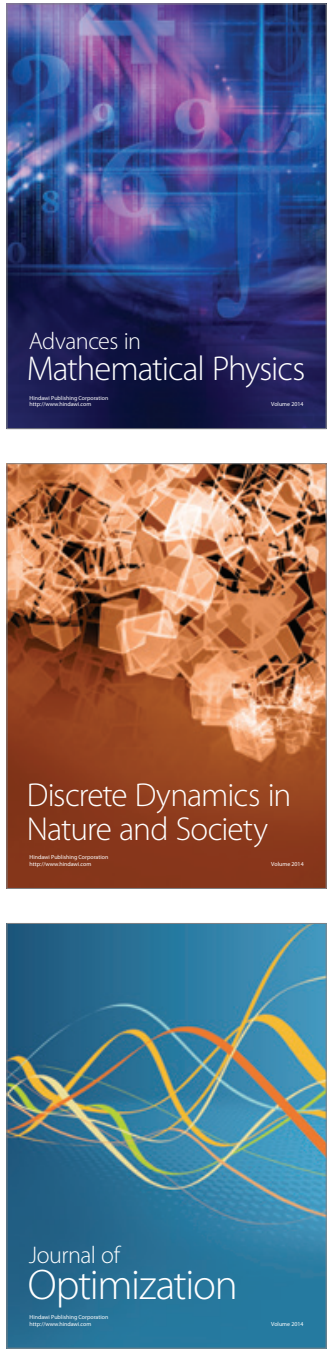